\title{
RELACIÓN DE LA POSTURA DE LA CABEZA Y EL ESPACIO FARÍNGEO EN PACIENTES CON MALOCLUSIÓN CLASE II ESQUELÉTICA
}

\author{
${ }^{1}$ Nhora Carolina Caraballo Aparicio, ${ }^{1}$ Bianca María Muré Mendoza, ${ }^{1}$ Thairy Auxiliadora Rangel Díaz
}

${ }^{1}$ Estudiantes de III año Especialización en Ortodoncia U. Santo Tomás, Bucaramanga (Colombia)

Autor responsable de correspondencia: Nhora Carolina Caraballo A.

Correo electrónico: nhoracarolina@hotmail.com

\begin{abstract}
RESUMEN
Objetivo: describir la relación de la postura de la cabeza con el espacio faríngeo en adultos mayores de 18 años con maloclusión Clase II esquelética por medio de análisis cefalométrico.

Materiales y métodos: se realizó un estudio descriptivo corte transversal, con una muestra conformada por 100 radiografias cefálicas laterales. Por medio del negatoscopio se visualizaron los respectivos puntos en las radiografias de perfil, para la realización del Cefalograma de Rocabado, donde se hicieron las mediciones de ángulos y planos, de ciertas estructuras óseas para ver las posibles alteraciones de acuerdo con valores estándares con el fin de tener en cuenta las repercusiones sobre el esqueleto craneofacial de los pacientes clase II.

Resultados: se evaluaron las radiografías cefálicas laterales de 100 personas quienes registraron una media de edad de 23 años \pm 5.6 ; el 63\% registraba un espacio faríngeo superior normal, el 54\% tenía una posición del hueso hioides neutra. El 55\% mostraba extensión del ángulo posteroinferior. El 59\% tenía una distancia CO - C1 normal. Hubo diferencias estadísticamente significativas al relacionar el ángulo posteroinferior con la edad categorizada.

Conclusiones: no se observaron asociaciones claras con las que se puedan establecer normas, entre la postura de la cabeza y el espacio faríngeo en los pacientes con maloclusión esquelética Clase II. [Caraballo NC, Muré BM, Rangel TA. Relación de la postura de la cabeza y el espacio faríngeo en pacientes con maloclusión clase II esquelética. Ustasalud 2011; 10: 103 - 109]

Palabras clave: Maloclusión Clase II esquelética, Postura de la cabeza, Espacio faríngeo, Cefalograma de Rocabado.

\section{RELATIONSHIP BETWEEN THE POSTURE OF THE HEAD AND THE PHARYNGEAL SPACE IN PATIENTS WITH SKELETAL CLASS II MALOCCLUSION}

\begin{abstract}
Objective: to establish the relationship between the posture of the head and the pharyngeal space in adults over 18 years with class II skeletal malocclusion using cephalometric analysis.

Methods: a cross sectional descriptive study was done with a sample of 100 lateral cephalic radiographs. The Rocabado's cephalogram was done in every radiograph to verify the possible changes in accordance with standard values in order know its impact on patients with craniofacial skeletal class II patients.

Results: the evaluation was done in lateral cephalic radiographs from 100 people who reported a mean age of $23 \pm 5.6,63 \%$ recorded a normal upper pharyngeal space, $54 \%$ had a neutral position of the hyoid bone. A posterior extension angle was showed in $55 \%$, $59 \%$ had a distance $\mathrm{CO}$ - C1 normal. There was a statistically significant difference between the posterior angle and the age categorized. Conclusions: there were no clear associations between the position of the head and pharyngeal space in patients with skeletal class II malocclusion.
\end{abstract}

Key words: Skeletal class II Malocclusion, Head posture, Pharyngeal space, Rocabado’s cephalogram.

Recibido para publicación: 12 de enero de 2011. Aceptado para publicación: 8 de mayo de 2011.

\section{INTRODUCCIÓN}

Los pacientes adultos con maloclusión clase II esquelética tienen diferentes características faciales y corporales que los llevan, por lo general, a presentar tanto problemas respiratorios como posturales. Esto ha sido confirmado por diversos autores como Rocabado y Solow que en algunos de sus estudios encontraron relación de la maloclusión Clase II con un ángulo craneofacial extendido o aumentado. ${ }^{1-4}$ Por otro lado, autores como Ceylan han visto que los pacientes con este tipo de maloclusión exhiben un espacio faríngeo más estrecho. ${ }^{5}$ Los ortodoncistas en el estudio diagnóstico cefalométrico que le deben realizar a sus pacientes tienden a sólo ver las características de la maloclusión sin tener en cuenta el estudio de la región cervical y del espacio faríngeo lo cual los llevaría a relacionar la maloclusión 
con la postura y la respiración respectivamente. Si se hace un análisis completo de los pacientes habida cuenta de todas estas características se podría brindar una atención más integral, en la que no sólo se corrija la posición de los dientes si no que se realice un tratamiento multidisciplinario que ayude a mejorar el nivel de vida de ellos. ${ }^{1-5}$

Una de las maloclusiones más difíciles de tratar y que causan una gran desarmonía facial son las maloclusiones clase II; en la cual el perfil retrognático y el resalte excesivo, exigen que la lengua se adapte a patrones anormales de contracción; y esto, en algunas ocasiones, puede disminuir la capacidad de crecimiento anteroposterior de la faringe lo cual puede producir obstrucción y disminución de la vía aérea, induciendo así una alteración en la posición de la mandíbula en reposo y una extensión de la espina cervical. Algunos autores ratifican la relación de la maloclusión clase II con un espacio faríngeo estrecho y encuentran que a medida que aumenta el ángulo ANB disminuye más este espacio, sin embargo, otros autores como Freitas no han encontrado que el tipo de maloclusión tenga influencia en el ancho del espacio faríngeo. ${ }^{5-8}$

La postura de la cabeza puede estar afectada por funciones como la audición, la vista, el equilibrio y algunas condiciones psicológicas, en adición con las funciones naso-respiratorias. Se ha demostrado que esta postura cráneo-cervical está relacionada con el desarrollo esquelético de la cara. Uno de los primeros en concluir que existía relación entre la postura de la cabeza, el desarrollo de las maloclusiones y la respiración fue Schwartz, él encontró que los pacientes con obstrucción de la vía aérea superior, presentaban una extensión extrema de la postura de la cabeza al dormir y tendían a desarrollar una maloclusión Clase II. La relación del aumento de la extensión de la cabeza con problemas respiratorios y con pacientes con maloclusión clase II, tanto adultos como niños, ha sido confirmada por otros autores como Wenzel, Ozbek y Miyamoto y Solow..$^{9-13}$

El objetivo de este estudio fue describir la postura de la cabeza y su relación con el espacio faríngeo superior e inferior, en pacientes con maloclusión clase II esquelética, mayores de 18 años, que asistieron a las Clínicas de Ortodoncia de la Facultad de Odontología de la Universidad Santo Tomás en Bucaramanga.

\section{MATERIALES Y MÉTODOS}

Se realizó un estudio observacional descriptivo de corte transversal; $;{ }^{14}$ el tamaño de la muestra estuvo compuesto por la totalidad de las radiografías cefálicas de las Historias Clínicas inactivas de las Clínicas de Ortodoncia de la Universidad Santo Tomás (Buca- ramanga). Entre los criterios de inclusión estaban las radiografias de pacientes con maloclusión clase II de tipo esquelético, mayores de 17 años, cuyas historias clínicas estuvieran inactivas y radiografías en buen estado. Se excluyeron las radiografias de pacientes que presentaran algún síndrome, pacientes con asimetrías o desviaciones esqueléticas y pacientes que hayan tenido tratamientos de ortodoncia u ortopedia. Las variables evaluadas fueron edad, género, triángulo hioideo, ángulo posteroinferior, distancia $\mathrm{CO}-\mathrm{C} 1$, espacio faríngeo superior, espacio faríngeo inferior.

Se tomaron los datos de las radiografias $(\mathrm{Rx})$ por medio de un negatoscopio, se visualizaron los respectivos puntos en las radiografias cefálicas laterales, para realizar el Cefalograma de Rocabado. La información fue digitalizada por duplicado en una base de datos en el programa Excel y validada en Epi info 6.04. Los datos fueron procesados en el programa Stata versión 9.0.

Para el análisis estadístico univariado se realizó una descripción de las características evaluadas mediante promedios y porcentajes según la naturaleza de las variables. En el bivariado llevado a cabo entre las variables que reflejaban la postura y las demás variables se aplicó el test Exacto de Fisher para las variables cualitativas y para las cuantitativas test de Anova o de Kruskal Wallis de acuerdo con la distribución de los datos.

Según la Resolución 008430 del Ministerio de Salud, esta investigación fue considerada sin riesgo, y fue sometida a revisión por parte del Comité de Ética de la Facultad de Odontología de la Universidad Santo Tomás en Bucaramanga.

\section{RESULTADOS}

\section{Análisis univariado}

Se evaluaron las radiografías de 100 personas quienes registraron una media de edad de 23 años \pm 5.6, 86 pertenecían al rango de edad entre 17 a 29 años, y 14 al rango de edad entre 30 a 46 años; el $72 \%$ (72) eran mujeres; el 63\% (63) registraban un espacio faríngeo superior normal (nasofaringe) y el $59 \%$ (59) un espacio faríngeo inferior (orofaringe) también normal, el 54\% (54) tenían una posición del hueso hiodes neutra (Tabla 1). El 55\% (55) extensión del Ángulo posteroinferior (Tabla 2). El 59\% (59) una distancia CO -C1 normal (Tabla 3).

\section{Análisis bivariado \\ Posición del hueso hioides}

Al analizar la posición del hueso hiodes con las variables sociodemográficas y el espacio faríngeo superior e inferior no se observaron asociaciones 
estadísticamente significativas; cabe destacar, sin embargo, que mientras que la mayoría de personas con posición del hueso hiodes negativa tenían el espacio faríngeo superior e inferior disminuido, el $6,3 \%$ (2) y el 5,9\% (2), respectivamente; la mayoría de personas con posición del hueso hiodes positiva y neutra tenían un espacio faríngeo superior normal, el $46 \%$ (29) y el 52,4\% (33), respectivamente. Así como un espacio faríngeo inferior normal, el $44,1 \%$ (26) y el $54,2 \%$ (32), respectivamente (Tabla 1).

\section{Ángulo posteroinferior}

Se encontraron diferencias estadísticamente significativas entre el ángulo posteroinferior y la edad categorizada, pero no se encontraron este tipo de asociaciones entre este ángulo y el espacio faríngeo superior e inferior; sin embargo, se puede destacar que la mayoría de pacientes con un ángulo en extensión, en posición normal y en flexión, presentan un espacio faríngeo superior normal, el 58,7\% (37), el 7,9\% (5) y el 33,3\% (21), respectivamente. En cuanto al espacio faríngeo inferior es posible resaltar que mientras que la mayoría de pacientes con extensión y flexión presentaban un tamaño normal de este espacio, el $54,2 \%$ (32) y el $40,7 \%$ (24) respectivamente; la mayoría de pacientes con un ángulo normal presentaban un tamaño del espacio faríngeo inferior disminuido, el $11,8 \%$ (4) (Tabla 2) (Figura 1).

\section{Distancia CO-C1}

Entre la distancia $\mathrm{CO}-\mathrm{C} 1$ y las variables sociodemográficas y el espacio faríngeo superior e inferior tampoco se encontraron asociaciones estadísticamente significativas, sin embargo, la mayoría de pacientes con una distancia $\mathrm{CO}-\mathrm{C} 1$ disminuida, normal y aumentada presentaron un espacio faríngeo superior normal, $12,7 \%$ (8), $60,3 \%$ (38) y $26,9 \%$ (17) respectivamente; así como un espacio faríngeo inferior normal, $13,6 \%$ (8), 50,9\% (30) y 35,6\% (21), respectivamente (Tabla 3 ).

\section{DISCUSIÓN}

En el presente estudio el objetivo era evaluar la postura en pacientes con maloclusión clase II esquelética, ya que existe la hipótesis de que estos pacientes presentan problemas posturales; esta situación ha sido confirmada por diferentes autores en sus numerosos estudios; además también se evaluó si estos problemas estaban relacionados con la disminución del espacio faríngeo tanto superior como inferior; debido a que los problemas posturales del cuerpo son un factor esencial en el desarrollo de las parafunciones orales, así como de otras funciones del sistema estomatognático, ya que las interconexiones nerviosas y anatómicas entre la cabeza y la columna cervical hacen que la posición alterada de una afecte a la otra. Aunque en los resultados no se observaron asociaciones claras con las que se puedan establecer normas, y sólo hubo un resultado estadísticamente significativo, si se muestran algunas tendencias que serían importantes para tener en cuenta en el análisis que se les realiza a los pacientes, para así poder brindar una mejor calidad de vida. ${ }^{15-17}$

En esta investigación se encontró que la mayoría de pacientes con maloclusión clase II presentaba una posición del hueso hiodes neutra (54\%), seguida de una posición positiva (43\%); mientras que la posición positiva representa normalidad en la ubicación del hueso hioides y en la postura; la posición neutra muestra alteración de esta, ya que aunque la relación craneovertebral pueda estar normal, hay pérdida de la curvatura cervical, lo que lleva a que el hueso hiodes se ubique en el plano retrognation - C3. Este resultado difiere al presentado en el estudio hecho por Henríquez y Ramón en adultos jóvenes mapuches sin disfunciones craneomandibulares, donde encontraron que el $60 \%$ presentaba una posición positiva y el $28,8 \%$ presentaba una posición neutra; esta diferencia se puede deber a que en este trabajo paciente si presentan una disfunción craneomandibular. La similitud entre estos estudios es que en los dos el menor porcentaje se dio en la posición del hueso hiodes negativa. ${ }^{18}$

$\mathrm{Al}$ relacionar la posición del hueso hiodes con el espacio faríngeo, fue evidente que los pacientes con una posición del hueso hiodes negativa (hueso por encima del plano retrognation - C3) presentaban mayor porcentaje de disminución del espacio faríngeo tanto superior como inferior; esto se puede deber a que la posición de este hueso estárelacionada con la respiración, una posición más baja del hueso hiodes (posición positiva o neutra) garantiza una buena respiración así como una buena fonación y deglución; sin embargo, existe la hipótesis que un mayor descenso de este hueso en la juventud o en la edad adulta puede estar relacionado con apnea obstructiva del sueño. Esta migración inferior del hueso hiodes es un fenómeno fisiológico, ya que los pacientes con problemas respiratorios mantienen su mandíbula en una posición descendida, situación que genera una disminución de la tensión en la musculatura suprahioídea y así el hioides es llevado hacia abajo; por lo tanto, esta posición más inferior del hueso también puede estar asociado con pacientes con retrognatismo mandibular y síntomas severos de asma, así como con pacientes dolicofaciales y adultos obesos, que por lo general presentan problemas respiratorios. ${ }^{19-22}$ 
Tabla 1. Descripción de las variables analizadas en forma global y según la posición del hueso hioides (triángulo hioideo)

\begin{tabular}{|c|c|c|c|c|c|}
\hline \multirow[b]{2}{*}{ Variable } & \multirow{2}{*}{$\begin{array}{l}\text { Global } \\
\text { n (\%) }\end{array}$} & \multicolumn{3}{|c|}{ Posición del hueso hiodes } & \multirow[b]{2}{*}{$\mathbf{p}$} \\
\hline & & $\begin{array}{c}\text { Positivo } \\
\text { n (\%) }\end{array}$ & $\begin{array}{l}\text { Neutra } \\
\text { n (\%) }\end{array}$ & $\begin{array}{c}\text { Negativo } \\
\text { n (\%) }\end{array}$ & \\
\hline Global & $100(100)$ & $43(43,0)$ & $54(54,0)$ & $3(3,0)$ & $\ldots$ \\
\hline Edad (años) & $23 \pm 5,6$ & $22,8 \pm 5,7$ & $23 \pm 5,6$ & $25 \pm 6$ & $\overline{0} 0 *$ \\
\hline 17 - 29 años & $86(86,0)$ & $36(41,9)$ & $42(54,7)$ & $3(3,5)$ & $0,8<0$ \\
\hline 30 - 46 años & $14(14,0)$ & $7(50,0)$ & $7(50,0)$ & --- & 0,856 \\
\hline \multicolumn{6}{|l|}{ Género } \\
\hline Hombres & $28(28)$ & $13(46,4)$ & $14(50,0)$ & $1(3,6)$ & \multirow[t]{2}{*}{0.984} \\
\hline Mujeres & $72(72)$ & $30(41,6)$ & $40(55,5)$ & $2(2,8)$ & \\
\hline \multicolumn{6}{|c|}{ Espacio Faríngeo Superior } \\
\hline Aumentado & $5(5)$ & $3(60,0)$ & $2(40,0)$ & --- & \multirow{3}{*}{0.323} \\
\hline Normal & $63(63)$ & $29(46,0)$ & $33(52,4)$ & 1 (1.6) & \\
\hline Disminuido & $32(32)$ & $11(34,4)$ & $19(59,4)$ & $2(6.3)$ & \\
\hline \multicolumn{6}{|c|}{ Espacio Faríngeo Inferior } \\
\hline Aumentado & $7(7,0)$ & $4(57,1)$ & $3(42,9)$ & --- & \multirow[t]{3}{*}{0.706} \\
\hline Normal & $59(59,0)$ & $26(44,1)$ & $32(54,2)$ & $1(1,7)$ & \\
\hline Disminuido & $34(34,0)$ & $13(38,2)$ & $19(55,9)$ & $2(5,9)$ & \\
\hline
\end{tabular}

Tabla 2. Descripción de las variables estudiadas analizadas en forma global y según el Ángulo Posteroinferior (API)

\begin{tabular}{|c|c|c|c|c|c|}
\hline \multirow[b]{2}{*}{ Variable } & \multirow[b]{2}{*}{$\begin{array}{l}\text { Global } \\
\text { n (\%) }\end{array}$} & \multicolumn{3}{|c|}{ Ángulo Posteroinferior API } & \multirow[b]{2}{*}{$\mathbf{P}^{*}$} \\
\hline & & $\begin{array}{c}\text { Extensión } \\
\text { n (\%) }\end{array}$ & $\begin{array}{c}\text { Normal } \\
\mathrm{n}(\%)\end{array}$ & $\begin{array}{c}\text { Flexión } \\
\text { n (\%) }\end{array}$ & \\
\hline Global & $100(100)$ & $55(55,0)$ & $9(9,0)$ & $36(36,0)$ & \\
\hline Edad (años) & $23 \pm 5,6$ & $24,2 \pm 6,4$ & $22,4 \pm 4,0$ & $21,5 \pm 3,9$ & --- \\
\hline 17-29 años & $86(86)$ & $42(48.8)$ & $9(10,5)$ & $35(40,7)$ & $0,074^{\text {** }}$ \\
\hline 30-46 años & $14(14)$ & $12(85.7)$ & --- & $2(14,3)$ & $0,045^{*}$ \\
\hline \multicolumn{6}{|l|}{ Género } \\
\hline Hombres & $28(28,0)$ & $15(53,6)$ & $2(7,1)$ & $11(39.3)$ & \multirow[t]{2}{*}{0,946} \\
\hline Mujeres & $72(72,0)$ & $40(55,6)$ & $7(9,7)$ & $25(34.7)$ & \\
\hline \multicolumn{6}{|c|}{ Espacio Faríngeo Superior } \\
\hline Aumentado & $5(5,0)$ & --- & $2(40,0)$ & $3(60,0)$ & \multirow{3}{*}{0,051} \\
\hline Normal & $63(63)$ & $37(58,7)$ & $5(7,9)$ & $21(33,3)$ & \\
\hline Disminuido & $32(32)$ & $18(56,3)$ & $2(6,3)$ & $12(37,5)$ & \\
\hline \multicolumn{6}{|c|}{ Espacio Faríngeo Inferior } \\
\hline Aumentado & $7(7)$ & $5(71,4)$ & $2(28,6)$ & --- & \multirow{3}{*}{0,084} \\
\hline Normal & $59(59)$ & $32(54,2)$ & $3(5,1)$ & $24(40,7)$ & \\
\hline Disminuido & $34(34)$ & $18(52,9)$ & $4(11,8)$ & $12(35,3)$ & \\
\hline
\end{tabular}

Test Exacto de Fisher

${ }^{* *}$ ANOVA $\quad \mathrm{p} \leq 0.05$

106 


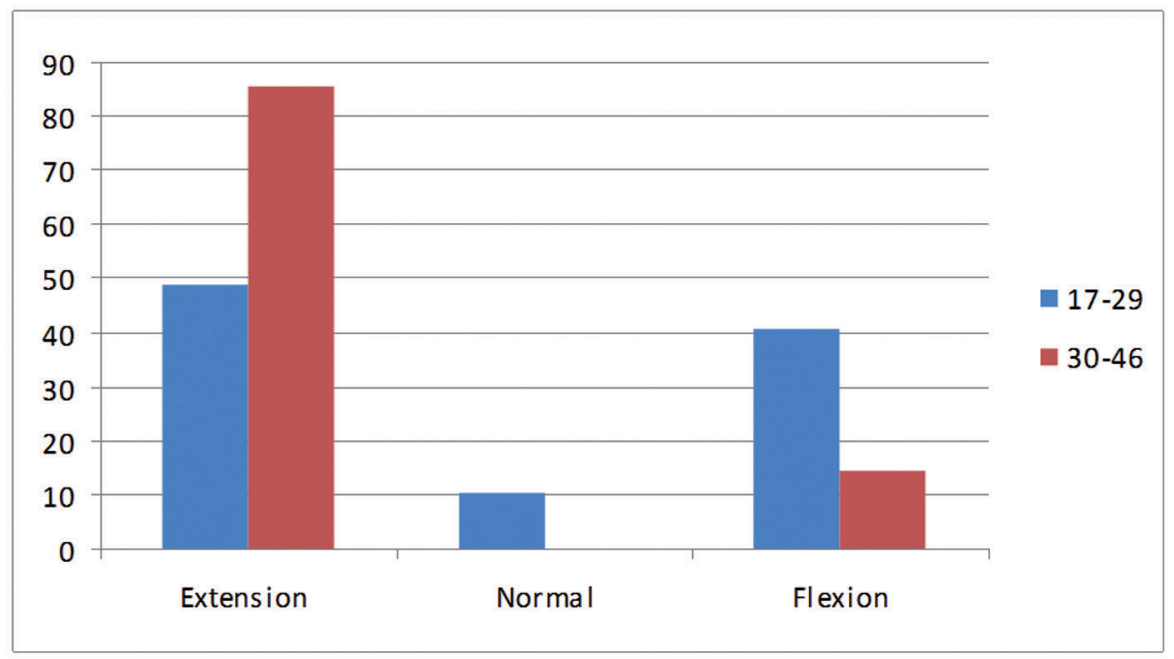

Figura 1. Relación entre el ángulo posteroinferior y la edad categorizada

Tabla 3. Descripción de variables estudiadas analizadas en forma global y según la Distancia CO-C1 (occipital y el arco posterior del atlas)

\begin{tabular}{|c|c|c|c|c|c|}
\hline \multirow[b]{2}{*}{ Variable } & \multirow[b]{2}{*}{$\begin{array}{l}\text { Global } \\
\text { n (\%) }\end{array}$} & \multicolumn{3}{|c|}{ Distancia CO - C1 } & \multirow[b]{2}{*}{$\mathbf{p}$} \\
\hline & & $\begin{array}{l}\text { Disminuida } \\
\text { n (\%) }\end{array}$ & $\begin{array}{c}\text { Normal } \\
\text { n (\%) }\end{array}$ & $\begin{array}{c}\text { Aumentada } \\
\text { n (\%) }\end{array}$ & \\
\hline Global & 100 & $12(12)$ & $59(59)$ & $29(29)$ & --- \\
\hline Edad (años) & $23 \pm 5,6$ & $24,3 \pm, 5,0$ & $23.4 \pm 6.1$ & $21,9 \pm 4,5$ & $0,359^{* *}$ \\
\hline 17- 29 años & $86(86)$ & $10(11,6)$ & 49 (57) & $27(31,4)$ & 0,453 \\
\hline 30 - 46 años & $14(14)$ & $2(14,3)$ & $10(71,4)$ & $2(14,3)$ & \\
\hline \multicolumn{6}{|l|}{ Género } \\
\hline Hombres & $28(28)$ & $4(14,3)$ & $13(46.4)$ & $11(39,3)$ & 0,260 \\
\hline Mujeres & $72(72)$ & $8(11,1)$ & $46(63.9)$ & $18(25)$ & \\
\hline \multicolumn{6}{|c|}{ Espacio Faríngeo Superior } \\
\hline Aumentado & $5(5)$ & --- & $2(40)$ & $3(60)$ & 0,729 \\
\hline Normal & $63(63)$ & $8(12.7)$ & $38(60.3)$ & $17(26,9)$ & \\
\hline Disminuido & $32(32)$ & $4(12.5)$ & 19(59.4) & $9(28,1)$ & \\
\hline \multicolumn{6}{|c|}{ Espacio Faríngeo Inferior } \\
\hline Aumentado & $7(7)$ & $1(14,3)$ & $4(57,1)$ & $2(28,6)$ & 0,269 \\
\hline Normal & 59 (59) & $8(13,6)$ & $30(50,9)$ & $21(35,6)$ & \\
\hline Disminuido & 34 (34) & $3(8,8)$ & $25(73,5)$ & $6(17,7)$ & \\
\hline
\end{tabular}

Test Exacto de Fisher $\quad{ }^{* *}$ ANOVA $\quad \mathrm{p} \leq 0.05$

En esta investigación no se podría determinar a pesar de que los pacientes son adultos con maloclusión clase II esquelética, si el porcentaje de estos que tuvieron una posición del hueso hiodes positiva (por debajo del plano retrognation - C3) esta relacionado con un patrón de normalidad en la postura o con una migración inferior del hueso asociada con apnea obstructiva del sueño, obesidad, problemas respiratorios como asma o con patrones dolicofaciales; ya que todos estos son aspectos que no se tuvieron en cuenta en el estudio.
El estudio del ángulo posteroinferior (API) se encontró que la mayoría de pacientes con maloclusión esquelética clase II (55\%) presentaban extensión de la cabeza; este resultado fue similar al del estudio realizado por Henríquez donde la mayoría de pacientes $(71,1 \%)$ presentaron también extensión de este ángulo; pero difiere del estudio hecho por Fuentes, donde la mayoría de pacientes adultos también sin ninguna disfunción craneomandibular como los pacientes estudiados por Henríquez, presentaron en su mayoría (60\%) un ángulo normal. ${ }^{18}$ 
Estos resultados contradictorios podrían indicar que no hay relación entre el tipo de maloclusión y el ángulo posteroinferior, esto puede estar asociado con lo que algunos autores como Villalón y colaboradores afirman, que este ángulo no representa una relación del cráneo con la columna cervical en su totalidad, sino más bien una relación del cráneo con la apófisis odontoides. Sin embargo, los resultados de este trabajo muestran una relación entre la maloclusión clase II y la extensión de la cabeza al igual que otros autores como Marcotte, Díaz, Sonnensen y D'Attilio quienes confirmaron esta asociación de una forma diferente a la de medir el ángulo posteroinferior. ${ }^{15,16,18,19,23-26}$

En esta investigación se encontró una diferencia estadísticamente significativa entre el ángulo posteroinferior y la edad categorizada ( $17-29$ y $30-46)$; esta diferencia mostró que es grande el porcentaje de personas entre 30 y 46 años con extensión de la cabeza, lo que puede ser debido a que después de los 20 años aumenta la longitud de la lengua y aumenta el grosor y la longitud del paladar blando lo que hace que disminuya el tamaño de la faringe, se reduce así la vía aérea y las personas adoptan una postura con extensión de la cabeza para mejorar el paso del aire. ${ }^{22,27}$

Al relacionar el ángulo posteroinferior con el espacio faríngeo no se encontró ningún patrón; debido a que, tanto en extensión, como en flexión y en un ángulo normal se encontró un tamaño del espacio faríngeo normal, excepto que para la mayoría de pacientes con un ángulo normal se evidenció una disminución del tamaño del espacio faríngeo inferior. Esto no tendría ninguna explicación, ni hay algún estudio que muestre esta misma relación; sin embargo, la explicación de que los pacientes con extensión presenten un espacio faríngeo normal, podría ser que, esta extensión de la cabeza produce un efecto favorable en la función nasorespiratoria. ${ }^{22,28}$

En cuanto a la medida de la distancia $\mathrm{CO}-\mathrm{C} 1$, se evidenció que la mayoría de pacientes (59\%) presentaban un tamaño normal de esta, seguido de un tamaño aumentado (29\%). Este resultado fue similar al del estudio de Fuentes, donde la mayoría de pacientes también presentaron una distancia normal $(51,8 \%)$, seguido de una distancia aumentada (33\%); pero, difiere al del estudio hecho por Henríquez donde la mayoría de pacientes presentó una distancia aumentada (51.1\%), seguido de una distancia normal (44.4\%). Sin embargo, llama la atención que en ninguno de estos estudios, incluido éste, se encontró una relación entre esta distancia y el ángulo posteroinferior como lo describe Rocabado; tampoco se halló que estas dos medidas fue- ran complementarias, ya que, por lo menos en este estudio mientras el ángulo posteroinferior indicaba para la mayoría de los pacientes una rotación posterior del cráneo, la distancia CO-C1 en la mayoría indicaba una normalidad en la posición del cráneo o una rotación anterior de éste; esto se puede deber a que las radiografias que se utilizaron para el estudio no fueron tomadas según el protocolo descrito por Rocabado. ${ }^{18,24,29}$

Al relacionar la distancia CO-C1 con el espacio faríngeo, se halló que todos los pacientes presentaron un espacio faríngeo superior e inferior normal, tanto en la distancia disminuida, normal, y aumentada; no se puede definir si hay alguna relación entre estas dos variables, y no hay ningún otro estudio donde las relacionen.

A pesar de que este trabajo se realizó en una muestra grande y en pacientes a los que no se les había realizado ningún tipo de tratamiento ortodóntico, presentó algunas limitaciones ya que no se tuvo en cuenta el efecto del género y algunos autores como Festa y Tecco han reportado dimorfismo sexual, manifiestan que el tamaño de las vértebras es mayor en hombres que en mujeres. Adicionalmente, otros autores ha reportado que en los hombres, a medida de que aumenta la edad, hay mayor disminución del espacio faríngeo que en las mujeres. ${ }^{27,30}$

Existe evidencia de que los pacientes con apnea obstructiva del sueño presentan problemas de postura como una posición más inferior del hueso hiodes, extensión de la cabeza y disfunciones craneomandibulares como retrognatismo inferior que esta relacionado con las maloclusiones clase II. Debido a que esta es una condición que afecta la respiración, existe la hipótesis de que haya alguna asociación clara entre la postura y el espacio faríngeo por lo menos para este tipo de pacientes, por lo que valdría la pena realizar este estudio en pacientes que presenten esta enfermedad. ${ }^{31,32}$

\section{Conclusiones}

No se observaron asociaciones claras con las que se puedan establecer normas, entre la postura de la cabeza y el espacio faríngeo en los pacientes con maloclusión esquelética clase II.

Mayor porcentaje de pacientes mayores de 30 años, con extensión craneocervical.

Gran parte de los pacientes presentaron una posición neutra del hueso hioides.

La distancia de $\mathrm{CO}-\mathrm{C} 1$ que predomino fue la normal.

El tamaño faríngeo superior e inferior fue normal en la mayoría de los pacientes del estudio. 


\section{BIBLIOGRAFÍA}

1. Rocabado M. Relaciones biomecánicas de las regiones craneales, cervicales e hiodeas bajo tratamiento ortodóntico y su incidencia con síntomas referidos. S.A.O 1994; 58: 59-63

2. Proffit W. Ortodoncia. Teoría y Práctica. 2da. Ed. Madrid: Mosby-Doyma Libros S.A.; 1996

3. Rocabado, M, Johnston BE, Blakney MG. Physical therapy and dentistry: An overview. J Craniomandibular Pract 1982; 1: 46 - 49

4. Solow B. Growth changes in head posture related to craniofacial development. Am J Orthod. 1986; 89: 132 - 140.

5. Ceylan I, Oktay H. A study on the pharyngeal size in different skeletal patterns. Am J Orthod Dentofacial Orthop 1995; 108: 69 - 75

6. Bishara, S. Ortodoncia. Ed Mc Graw Hill: México D.F.: 2003

7. Tourné L. Growth of the pharynx and its physiologic implications. Am J Orthod Dentofacial Orthop 1991; 99: $129-139$

8. Freita M, Penteado N. Upper and lower pharyngeal airways in subjects with class I and class II malocclusions and different growth patterns. Am J Orthod Dentofac Orthop 2006; 130: 742 - 745

9. Solow B. Tallgren, A. Head posture and craniofacial morphology. Am J Phys Anthropol 1976; 44: 417 - 436

10. Solow B. Sonnesen L. Head Posture and malocclusion. Eur J Orthod 1998; 20: 685 - 693

11. Wenzel, A. Craniofacial morphology and head posture in children with asthma and perennial rhinitis. Eur J Orthod 1985; 7: $83-89$

12. Ozbek M, Miyamoto K. Natural head posture, upper airway morphology and obstructive sleep apnea severity in adults. Eur J Orthod 1998; 20: 133 - 143

13. Solow, B. Ovesen, J. Head posture in obstructive sleep apnea. Eur J Orthod 1993; 15: 107-114

14. Pineda E, Alvarado E, Canales F. Metodología de la Investigación. Segunda Edición. Washington, DC: Organización Panamericana de la Salud; 1994

15. Diaz M. Estudio de las vértebras cervicales en pacientes con maloclusiones usando la posición natural de la cabeza. Revista Facultad de Odontología de la Universidad de Carabobo; 2002

16. Sonnesen L, Kjaer I. Anomalies of the cervical vertebrae in patients with skeletal class II malocclusion and horizontal maxillary overjet. Am J Orthod Dentofacial Orthop 2008; 133: e15 - e20

17. Amis T $\mathrm{O}^{\prime} \mathrm{Neill} \mathrm{N}$. Oral airway flow dynamics in healthy humans. J Physiol 1999; 515: 293 -298

18. Henríquez J, Ramón F. Análisis de la estabilidad ortoestática craneocervical en adultos jóvenes mapuches. Int J Morphol 2003; 21: 149 - 153

19. Lieberman D, McCarthy R, Hiiemae K. Odontogeny of postnatal hyoid and larynx descent in humans. Arch Oral Biol 2001; 46: 117 - 128

20. Worsnop C, Kay A, Kim Y. Effect of age on sleep onsetrelated changes in respiratory pump and upper airway muscle function. J Appl Physiol 2000; 88: 1831 - 1839

21. Pae E, Quas C, Quas J. Can facial type be used to predict changes in hyoid bone position with age? A perspective based on longitudinal data. Am J Orthod Dentofacial Orthop 2008; 134: $792-797$

22. González A, Manns A. Forward head posture: It's structural \& functional influence on the stomatognatic system, a conceptual study. J Craniomandibular Prac 1996; 14: $77-80$

23. Marcotte M. Head posture and dentofacial proportions. Angle Orthod 1981; 51: $208-213$

24. Fuentes R, Henriquez J, Sandoval P, Matamala F. Estudio anátomo-radiológico de la región craneocervical en 60 estudiantes de la Universidad de la Frontera. Rev Med Chile 1996; 124: 1483 - 1488

25. Villalón P, Frugone R, Palomino H. Algunas mediciones radiográficas cráneo-cervicales según biotipo de Ricketts. Revista Dental de Chile 2004; 95: 11 - 17

26. D'Attilio M, Caputi S, Epifania E, Festa F, Tecco S. Evaluation of cervical posture of children in skeletal class I, II and III. Cranio 2005; 23: 219 - 228

27. Kollias L, Krogstad O. Adult craniocervical and pharyngeal changes a longitudinal cephalometric study between 22 and 42 years of age. Part II: morphological uvulo-glossopharyngeal changes. Eur J Orthod 1999; 21: $345-355$

28. Huggare J. Nasorespiratory function and head posture. Am J Orthod Dentofacial Orthop 1997; 112: 507 - 511

29. Rocabado M. Análisis biomecánico cráneocervical a través de una teleradiografía lateral. Rev Chilena de Ortodoncia 1984; $1: 42-52$

30. Festa F, Tecco S. Relationship between cervical lordosis and facial morphology in Caucasian women with a skeletal class II malocclusion: a cross-sectional study. Cranio 2003; $21: 121-129$

31. Ozbek, M. Miyamoto, K. Natural head posture, upper airway morphology and obstructive sleep apnea severity in adults. Eur J Orthod 1998; 20: 133 - 143

32. Tangugsorn V, Krogstad O, Espeland L, Lyberg T. Obstructive sleep apnoea: multiple comparisons of cephalometric variables of obese and non-obese patients. J Cranio Maxillofacial Surg 2000; 28: $204-212$

\section{Correos electrónicos de los autores:}

Nhora Carolina Caraballo A.: nhoracarolina@hotmail.com Bianca María Mure Mendoza: biancamure@hotmail.com Thairy A Rangel Díaz: thairyrangel@hotmail.com 